\title{
BLEOMYCIN-INDUCED REVERSIBLE ACUTE INTERSTITIAL PNEUMONIA
}

\author{
BALAJI 0 ${ }^{1}$, AMITA D ${ }^{1}$, NAVIN PATIL ${ }^{1 *}$, JOSEPH THOMAS ${ }^{2}$
}

${ }^{1}$ Department of Pharmacology, Kasturba Medical College, Manipal, Karnataka, India. ${ }^{2}$ Department of Medical Oncology, Kasturba Medical College, Manipal, Karnataka, India. Email: navin903@gmail.com

Received: 16 January 2017, Revised and Accepted: 28 February 2017

ABSTRACT

As of now, about 380 medications are implicated in causing respiratory reactions and most common among that is drug-induced interstitial disease. Oral, parental as well inhalational drugs are known to cause drug-induced interstitial lung disease. Bleomycin is a chemotherapeutic agent used in the treatment of lymphomas, germ cell tumors of the testes. Most common pulmonary toxicity is diffuse alveolar damage with nonspecific interstitial pneumonitis being next. We report a case of bleomycin-induced reversible acute interstitial pneumonia in a Hodgkin's lymphoma patient with adriamycin, bleomycin, vinblastine, dacarbazine regimen. Causality assessment was done using Naranjo scale, and probable causal relationship was established. Adverse drug reaction was found to be moderately severe and not preventable as per Hartwig's severity and Thornton's preventability scaling respectively.

Keywords: Naranjo scale, Lymphoma, Bleomycin, Pneumonia.

(c) 2017 The Authors. Published by Innovare Academic Sciences Pvt Ltd. This is an open access article under the CC BY license (http://creativecommons. org/licenses/by/4. 0/) DOI: http://dx.doi.org/10.22159/ajpcr.2017.v10i5.17121

\section{INTRODUCTION}

The lungs, with respect to the large surface area, are a potential target for many dreaded toxic substances. Specific respiratory reactions are noted in lungs as a part of generalized reactions which may be because of disease per se or may be because of substances such as drugs, toxins and the rest. Lungs are one of the major sites for metabolism of drugs. As of now, about 380 medications are implicated in causing respiratory reactions and most common among that is drug-induced interstitial disease [1]. Other includes involvement of pleura, parenchyma, airways, mediastinum, and pulmonary vasculature. Oral, parental as well inhalational drugs are known to cause drug-induced interstitial lung disease (DILD) [2]. Bleomycin is a chemotherapeutic agent used in the treatment of lymphomas, germ cell tumors of the testes. Most common pulmonary toxicity is diffuse alveolar damage with nonspecific interstitial pneumonitis (NSIP) being next [3]. Hence forth, we report a case of bleomycin-induced reversible acute interstitial pneumonia in a Hodgkin's lymphoma patient with adriamycin, bleomycin, vinblastine, dacarbazine regimen (ABVD regimen).

\section{CASE REPORT}

Informed consent was taken from the patient. A 43-year-old male came with complaints of abdominal pain of insidious onset, progressive, nonradiating since 1 month duration. It was not associated with altered bowels, fever, vomiting, melena, hematemesis, and jaundice. Vitals were stable, bowel sounds were normal, and no organomegaly was noted. Other system examination was normal. Computed tomography (CT) scan of abdomen showed enlarged homogenously enhancing abdominal lymph nodes conglomerated in para-aortic, aortocaval, periportal, and peripancreatic region (Fig. 1). Diagnostic laparoscopy was done to differentiate between abdominal tuberculosis and lymphoma, biopsy of para-aortic nodes revealed Hodgkin's lymphoma of nodular sclerosis type. CT scan of chest was taken to rule out metastasis and it was normal (Fig. 2). Blood, liver and renal function tests were normal. Hence, the patient was started on adriamycin $35 \mathrm{~g}$, bleomycin $14 \mathrm{mg}$, vinblastine $8.4 \mathrm{mg}$, dacarbazine $530 \mathrm{mg}$ (ABVD regimen).

The first cycle was completed, and the patient was fine. Six cycles were given every fortnight. On the end of sixth cycle patient complained of fever, cough, grade 2 dyspnea, and blue discoloration of nails. Possibility of opportunistic infections was ruled out as sputum and respiratory culture was negative. Chest X-ray revealed inhomogeneous fluffy opacities in right upper and the middle lobe. Hence, CT was taken and it showed diffuse ground glass opacification involving both lungs $(\mathrm{R}>\mathrm{L})$ predominantly in the perihilar region and right middle and lower lobes, mild interlobular septal thickening in the basal segments of the right lower lobe and medial and lateral segment of right middle lobe, mild cystic and cylindrical bronchiectasis changes as described. Possibility of diffuse alveolar injury/acute interstitial pneumonia secondary to bleomycin drug-induced toxicity (Fig. 3). Follow-up image after 10 days revealed features consistent with NSIP pattern confirming the diagnosis of drug-induced incidence of ILD.

Hence, the patient was started immediately with levofloxacin $750 \mathrm{mg}$ twice daily for 7 days, wysolone $30 \mathrm{mg}$ once daily for 10 days, and tablet acyclovir $800 \mathrm{mg}$ thrice daily for 5 days. Patient condition improved and repeats CT scan showed minimal bronchiectatic changes with a reduction in ground glass opacity signifying reversal of interstitial pneumonia (Fig. 4). Later wysolone was slowly tapered over 10 days, and bleomycin was removed from the treatment regimen and patient continued with his chemotherapy.

\section{DISCUSSION}

It is being reported that 2 million cases of adverse drug reactions occur every year in the United States, including 1,00,000 deaths. Lungs being a major site affected by drugs up to $10 \%$ adverse drug reactions result in pulmonary toxicity. Literate predicts this number might escalate further as DILD is underdiagnosed worldwide and also due to the advent of newer medications. ILD worldwide is not clearly known but in 2.5-3\% drugs have been implicated as the causative factor. Risk factor for DILD includes cancer medications, patients treated for rheumatoid arthritis, extremes of age, pre-existing lung disease, ethnicity, genetic polymorphisms, drug interactions, and radiation.

Most common drugs causing pulmonary toxicity include cytotoxic agents, namely, carmustine, cyclophosphamide and bleomycin, amiodarone, aspirin, nitrofurantoin, sulfonamides, amphotericin B, biological agents, and other miscellaneous drugs. Mechanisms put forth in DILD includes injury to alveolar and bronchial epithelial due to specificity of some substances, direct toxicity in case of 


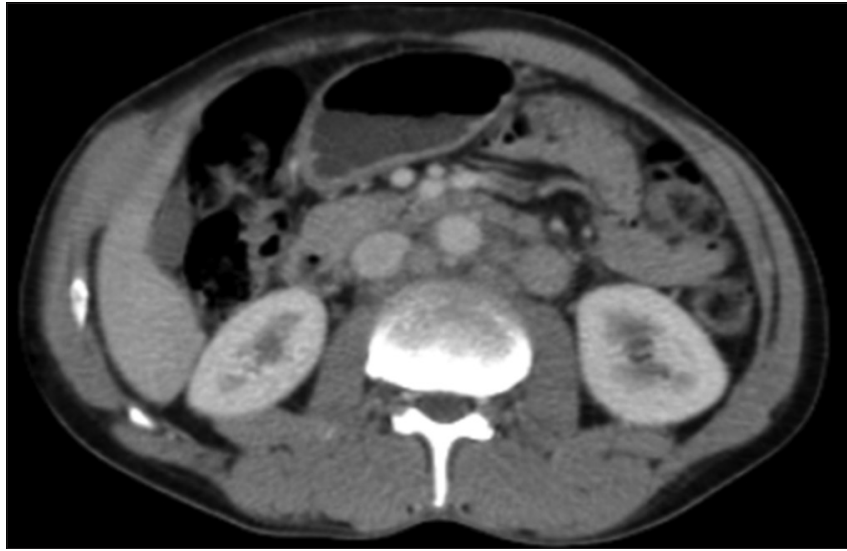

Fig. 1: Computed tomography scan abdomen with enlarged lymph nodes in para-aortic, aortocaval, periportal, and peripancreatic region

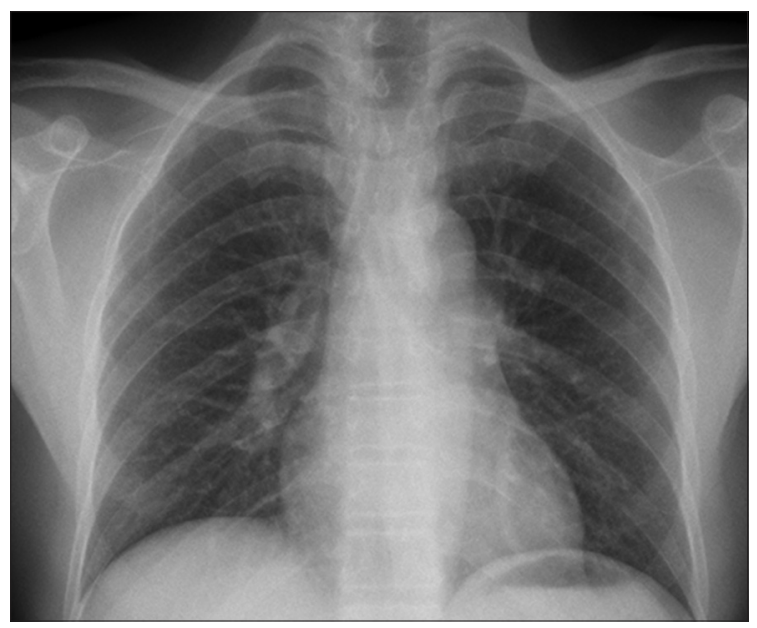

Fig. 2: Normal chest X-ray before chemotherapy

chemotherapeutic agents, due to inadequate removal toxic metabolites, disturbance in oxidant/antioxidant hemostasis, due to immune medicated antigen - antibody complex deposition and finally due to aggregation of amphiphilic compounds in alveolar macrophages [4].

Bleomycin, an antibiotic, isolated from the fungus streptomyces verticillus with antitumor activity was discovered by umezewa in 1966. Bleomycin exerts its antitumor effect by inducing tumor cell death, while inhibition of tumor angiogenesis may also be important. $\mathrm{ABVD}$ and bleomycin, etoposide, cisplatin are common regimens that include bleomycin for treatment of Hodgkin's lymphoma and germ cell tumor, Kaposi's sarcoma, cervical cancer, and squamous cell carcinomas of the head and neck are other tumors where bleomycin is used. Recently, bleomycin is being used in percutaneous sclerotherapy of venous malformations and lymphangiomas. Bleomycin is used in combination chemotherapy regimens because of its broad activity and low myelotoxicity [5].

Bleomycin, a G2 and M phase cell cycle specific agent, with a molecular weight of 1500 has two binding regions, one for iron and other for DNA. Cytotoxic activity of bleomycin is mainly because of iron being an essential cofactor. Free radical that causes breaks in DNA single and double strands are formed when bleomycin binds to iron and oxidizes iron molecule finally culminating in cell death. It is said to cause oxidative degradation of cellular RNA as well. It has a half-life of $3 \mathrm{hrs}$, and enzyme bleomycin hydrolase rapidly inactivates it in the liver and kidneys. Since $60-70 \%$ of drug is eliminated via kidneys careful monitoring and dose reduction is required in case of renal dysfunction. Side effects profile

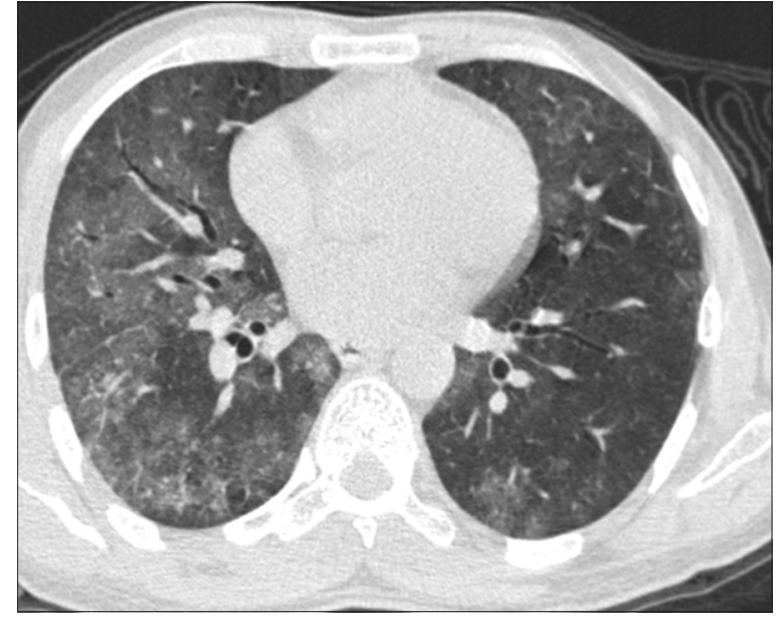

Fig. 3: Computed tomography chest showing acute interstitial pneumonia

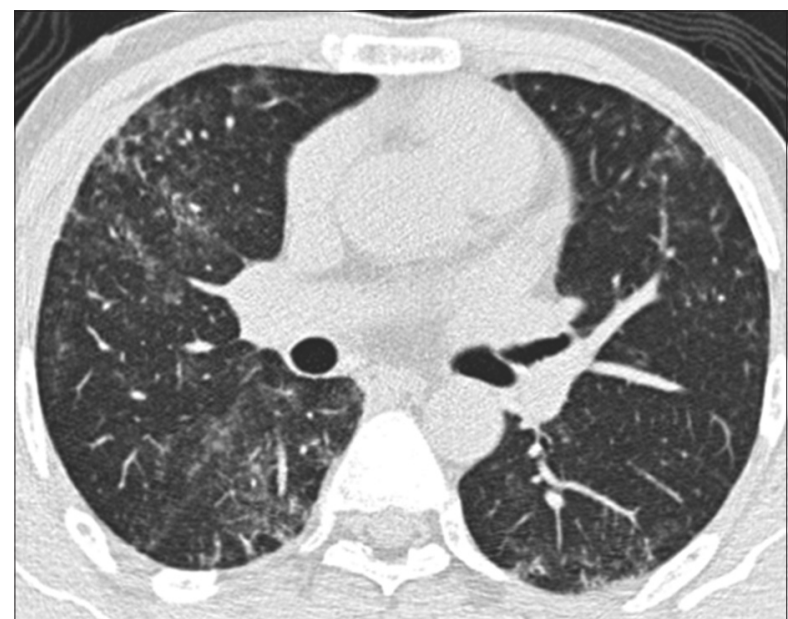

Fig. 4: Repeat computed tomography chest showing reversal of acute interstitial pneumonia

includes erythema, hyperpigmentation of the skin, discoloration of nail bed, hyperkeratosis, chills, hypotension, alopecia, stomatitis, myocardial infarction, stroke, and Raynaud's phenomenon [6].

One of the major limitations of bleomycin therapy is pulmonary toxicity. It can occur up to $10 \%$ since lungs have very less amounts of bleomycin hydrolase required for metabolizing bleomycin into nontoxic metabolites. Pulmonary syndromes associated with bleomycin includes bronchiolitis obliterans organizing pneumonia, eosinophilic hypersensitivity, and with most common being interstitial pneumonitis. The latter, bleomycin-induced pneumonitis (BIP) has an incidence ranging from $0 \%$ to $46 \%$ with a reasonable estimate of BIP incidence being $10 \%$ [6]. Mortality ranges up to $10 \%$ [7]. Common symptoms include dyspnea, clubbing, nonproductive cough, pneumonitis, tachypnea, cyanosis with end-inspiratory bibasilar crepitation's, and pleural rubbing.

Pathogenesis involves oxidative damage, deficiency of the bleomycin hydrolase, genetic susceptibility, and cytokine-mediated inflammation. Membrane instability and inflammation due to fatty acid oxidation occurs due to free radical formation, cytokines such as interleukin-1, macrophage inflammatory protein-1, platelet-derived growth factor, and Transforming growth factor-beta are released from alveolar macrophages and this further causes proliferation of fibroblasts leading to pulmonary fibrosis. Other postulated mechanisms include loss of pulmonary immune tolerance, disruption of balance between collagen 
Table 1: Adverse drug reaction scaling and assessment

\begin{tabular}{ll}
\hline Scaling & Assessment \\
\hline Naranjo & Probable \\
Hartwig's & Moderately severe \\
Thornton's & Not preventable \\
\hline
\end{tabular}

deposition and collagenolysis as well fibrin and fibrinolytic system resulting in excess collagen and fibrin deposition, respectively [6]

Diagnosis of DILD is based on following criteria [4], (1) a history of drug exposure with correct identification of the drug, its dose, and its duration of administration, (2) clinical, imaging, and histopathological patterns which are consistent with earlier observations with the same drug, (3) exclusion of other lung disease, (4) improvement following discontinuation of the suspected drug, and (5) recurrence of symptoms on rechallenge, but rechallenge can be hazardous.

In our case with the history of exposure to bleomycin other possible conditions mimicking DILD was excluded which includes Pneumocystis jiroveci as its more common in patients with Hodgkin's lymphoma on chemotherapy and other bacterial, viral infections. CT imaging was done to confirm the diagnosis, dechallenge was also done and symptoms improved with treatment and repeat CT scan was taken to confirm reversal of DILD. Causality assessment was done using Naranjo scale, and probable causal relationship was established [8]. Adverse drug reaction was found to be moderately severe and not preventable as per Hartwig's severity [8] and Thornton's preventability scaling [8] respectively (Table 1 ).

\section{REFERENCES}

1. Flieder DB, Travis WD. Pathologic characteristics of drug-induced lung disease. Clin Chest Med 2004;25(1):37-45.

2. Nemery B, Bast A, Behr J, Borm PJ, Bourke SJ, Camus PH, et al. Interstitial lung disease induced by exogenous agents: Factors governing susceptibility. Eur Respir J Suppl 2001;32:30s-42.

3. Rossi SE, Erasmus JJ, McAdams HP, Sporn TA, Goodman PC. Pulmonary drug toxicity: Radiologic and pathologic manifestations. Radiographics 2000;20(5):1245-59.

4. Schwaiblmair M, Behr W, Haeckel T, Märkl B, Foerg W, Berghaus T. Drug induced interstitial lung disease. Open Respir Med J 2012;6:63-74.

5. Harani M, Kalav SV. Study on the protective efficacy of Brassica rapa chinensis against bleomycin induced pulmonary fibrosis. Int J Pharm Pharm Sci 2014;6(5):53-8.

6. Reinert T, Baldotto CS, Nunes FA, Scheliga AA. Bleomycin-induced lung injury. J Cancer Res 2013;2013:9.

7. Patil N, Paulose RM, Udupa KS, Ramakrishna N, Ahmed T. Pulmonary toxicity of bleomycin - A case series from a tertiary care center in Southern India. J Clin Diagn Res 2016;10(4):FR01-3.

8. Balaji O, Navin P, Avinash A, Amod T. Cefuroxime-induced thrombocytopenia: It's just not in the ring? Asian J Pharm Clin Res $2016 ; 9(5): 1-2$ 